\title{
Ultra-Wideband Calibration for Indoor Positioning
}

\author{
Jeroen D. Hol*t, Thomas B. Schön ${ }^{\dagger}$ and Fredrik Gustafsson ${ }^{\dagger}$ \\ *Xsens Technologies B.V., Enschede, The Netherlands \\ ${ }^{\dagger}$ Division of Automatic Control, Linköping University, Sweden
}

\begin{abstract}
The main contribution of this work is a novel calibration method to determine the clock parameters of the UWB receivers as well as their $3 D$ positions. It exclusively uses time-of-arrival measurements, thereby removing the need for the typically labor-intensive and time-consuming process of surveying the receiver positions. Experiments show that the method is capable of accurately calibrating a UWB setup within minutes.

Index Terms-Calibration, maximum likelihood estimation, ultra-wideband, indoor positioning.
\end{abstract}

\section{INTRODUCTION}

Ultra-wideband (UWB) is a relatively new and promising localization technology, especially for indoor applications. Among its more mature applications are the so-called asset tracking systems in for instance health-care or manufacturing. Commercially available systems [1,2] typically consist of a network of synchronized UWB receivers which track a large number of small, battery powered and inexpensive UWB transmitters. Reported indoor position accuracies lie in the order of decimeters, but suffer from multipath effects and nonline-of-sight (NLOS) conditions.

The trilateration problem, i.e., determining the position of a transmitter, is a well-studied topic for which many algorithms are reported in literature, see for instance [3, 4, 5, 6]. Although a correct calibration of the setup is a prerequisite for accurate positioning results, it is typically taken for granted and calibration is very seldom discussed. To the best of the authors knowledge, current calibration methods have been developed by the hardware manufacturers and are documented in their manuals. These methods require receiver positions and transmitter positions to be surveyed. The surveying of positions typically is a time-consuming and error-prone process which requires additional equipment. It is only feasible for permanent setups, which severely limits the deployment of UWB positioning systems. The main contribution of this paper is a flexible and easy-to-use calibration algorithm capable of calibrating a UWB setup without additional measurements and hardware in a couple of minutes, thereby enabling flexible and portable UWB positioning systems.

\section{Problem Definition}

Ultra-wideband technology makes use of radio with very short pulses, typically in the order of $1 \mathrm{~ns}$, resulting in a high spatial resolution. The positioning technologies can roughly be subdivided into three categories: systems using time delay, systems using angle-of-arrival and systems using signal strength [4]. In this paper we focus on time-based methods, where position is inferred from the time it takes for a signal to travel from the transmitter to the receiver.

The UWB setup consists of a network of synchronized and stationary (rigidly fixed, mounted) receivers, all taking very precise time-of-arrival (TOA) measurements of signals originating from a transmitter. That is, the TOA measurement $y_{m n k}$ is the time (according to its local clock) when receiver $m$ receives the $k$-th pulse from transmitter $n$. It can be modeled as

$$
y_{m n k}=\tau_{n k}+\left\|\boldsymbol{r}_{m}-\boldsymbol{t}_{n k}\right\|_{2}+\Delta \tau_{m}+\delta_{m n k}+e_{m n k},
$$

where $\tau_{n k}$ is the time-of-transmission of the $k$-th pulse from transmitter $n, \boldsymbol{t}_{n k}$ is the position of the transmitter of the $k$ th pulse from transmitter $n, \boldsymbol{r}_{m}$ is the position of the $m$-th receiver and $\Delta \tau_{m}$ is the clock-offset of the $m$-th receiver. $\delta_{m n k}>0$ is a possibly nonzero delay due to non line-of-sight conditions or multipath and $e_{m n k}$ is Gaussian noise. Without loss of generality, all quantities in (1) are expressed in meters.

The experimentally observed measurement noise is virtually independent of range, contrary to what typically is observed for signal strength measurements, hence we assume $e_{m n k}$ to be independent identically distributed (i.i.d.) Gaussian noise, that is, $e_{m n k} \sim \mathcal{N}\left(0, \sigma^{2}\right)$. In the remainder of this paper we assume 'clean' measurements, i.e., $\delta_{m n k}=0$. The subscripts $m, n$ and $k$ will be dropped when their value can be inferred from the context. The transmitter clocks are not sufficiently accurate to know their precise time-of-transmissions $\tau$ and since only oneway communication is possible they cannot be synchronized to the receiver clocks. Therefore, every time-of-transmission $\tau$ has to be treated as an unknown parameter. This makes the UWB measurements very similar to GPS pseudo-ranges [7].

The process of determining the transmitter position from the UWB measurements is referred to as trilateration or multilateration, for which several approaches can be found in the literature. A common technique is to eliminate the timeof-transmission $\tau$ from (1) by constructing time difference of arrival (TDOA) measurements from pairs of measurements. The resulting set of hyperbolic equations can then be efficiently solved for position [3,5]. The drawback of this approach is that the constructed TDOA measurements are no longer independently distributed. An alternative, but equivalent approach is to treat $\tau$ as an unknown and solve for both position and time. Assuming Gaussian noise, maximum likelihood estimation takes the form of a nonlinear least squares problem,

$$
\min _{t, \tau} \sum_{m=1}^{M}\left(y_{m}-\tau-\left\|\boldsymbol{r}_{m}-\boldsymbol{t}\right\|_{2}-\Delta \tau_{m}\right)^{2},
$$

which can be efficiently solved using standard algorithms from nonlinear numerical optimization $[8,9]$.

Note that the trilateration procedure (2) assumes known values for the receiver parameters $r$ and $\Delta \tau$. In this paper we will derive an algorithm that is capable of estimating these parameters using a maximum likelihood approach. That is, we 
want to estimate the $3 \mathrm{D}$ receiver positions $\left\{\boldsymbol{r}_{m}\right\}_{m=1}^{M}$ as well as the receiver clock-offsets $\left\{\Delta \tau_{m}\right\}_{m=1}^{M}$ for all $M$ receivers.

\section{CAlibration}

In this section we describe an existing calibration method and show how we extend it to a flexible and easy-to-use calibration algorithm.

\section{A. Existing methods}

The current state-of-the-art calibration methods focus on estimating the receiver clock-offset $\Delta \tau_{m}$. They require a dataset consisting of $(K)$ transmissions from one or more $(N \geq 1)$ transmitters at known positions $\overline{\boldsymbol{t}}_{n}$ to a number of $(M)$ receivers, also at known positions $\overline{\boldsymbol{r}}_{m}$. Given $\overline{\boldsymbol{t}}_{n}$ and $\overline{\boldsymbol{r}}_{m}$, the clock-offset differences $\Delta \tau_{m}-\Delta \tau_{1}$ are straightforwardly given from (averaged) TDOA measurements,

$$
\begin{aligned}
z_{m n k}= & y_{m n k}-y_{1 n k}=\left\|\boldsymbol{r}_{m}-\boldsymbol{t}_{n k}\right\|_{2} \\
& -\left\|\boldsymbol{r}_{1}-\boldsymbol{t}_{n k}\right\|_{2}+\Delta \tau_{m}-\Delta \tau_{1}+v_{m n k},
\end{aligned}
$$

since the ranges are known and can be eliminated. Slightly more advanced methods use multiple tags and estimate the clock-offset taking into account the correlated noise. The downside of this algorithm is that all involved positions have to be specified, and surveying them typically is a labor-intensive and time-consuming process.

The above procedure can be formulated as a constrained maximum likelihood (ML) problem. To do so, we first define the parameter vector $\boldsymbol{\theta}$ as

$$
\boldsymbol{\theta}=\left(\left\{\boldsymbol{t}_{n},\left\{\tau_{n k}\right\}_{k=1}^{K}\right\}_{n=1}^{N},\left\{\boldsymbol{r}_{m}, \Delta \tau_{m}\right\}_{m=1}^{M}\right) .
$$

Since we assume Gaussian measurement noise, the probability density function of the measurements is given as

$$
p\left(y_{m n k} ; \boldsymbol{\theta}\right)=\frac{1}{\sqrt{2 \pi \sigma^{2}}} \exp \left(-\frac{1}{2} \epsilon_{m n k}^{2}(\boldsymbol{\theta})\right),
$$

where according to (1) the normalized residuals $\epsilon_{m n k}$ are given as

$$
\epsilon_{m n k}(\boldsymbol{\theta})=\sigma^{-1}\left(\tau_{n k}+\left\|\boldsymbol{r}_{m}+\boldsymbol{t}_{n}\right\|_{2}+\Delta \tau_{m}-y_{m n k}\right) .
$$

Using (5) and (6), the maximum likelihood problem for estimating $\boldsymbol{\theta}$ becomes

$$
\begin{array}{ccc}
\min _{\boldsymbol{\theta}} & \frac{1}{2} \sum_{m=1}^{M} \sum_{n=1}^{N} \sum_{k=1}^{K} \epsilon_{m n k}^{2}(\boldsymbol{\theta}) \\
\text { s.t. } & \boldsymbol{t}_{n}-\overline{\boldsymbol{t}}_{n}=0 \quad n=1, \ldots, N \\
& \boldsymbol{r}_{m}-\overline{\boldsymbol{r}}_{m}=0 \quad m=1, \ldots, M \\
\Delta \tau_{1}=0 &
\end{array}
$$

The constraints (7b)-(7d) specify the surveyed positions of the receivers and the transmitters, and without loss of generality the clock of the first receiver is selected to be the central clock. The latter is necessary since a common constant can be added to the clock-offsets and subtracted from the timeof-transmissions without affecting the normalized residuals. Note that although the problem (7) is formulated using a large parameter vector, the constraints $(7 \mathrm{~b})$ and $(7 \mathrm{c})$ reduce it to finding $\left\{\tau_{n k}\right\}$ and $\left\{\Delta \tau_{m}\right\}$. The above derivation (5)-(7) can equivalently be derived for TDOA measurements, resulting in a weighted objective for (7a).

\section{B. Proposed method}

To arrive at our proposed calibration method, note that the constraints (7b) and (7c) are not essential to solve the problem and can be removed. Hence, it is possible to estimate the complete parameter vector $\boldsymbol{\theta}$, including the receiver and tag positions, from the dataset $\left\{y_{m n k}\right\}$ and skip the surveying step completely. Similarly to selecting a central clock, we have to define the coordinate system in which the positions are expressed. Without loss of generality, we define it using three (non-collinear) receivers. Summarizing the above, we propose to formulate the calibration problem as

$$
\begin{array}{ll}
\min _{\boldsymbol{\theta}} & \frac{1}{2} \sum_{m=1}^{M} \sum_{n=1}^{N} \sum_{k=1}^{K} \epsilon_{m n k}^{2}(\boldsymbol{\theta}) \\
\text { s.t. } & A_{m} \boldsymbol{r}_{m}=\mathbf{0} \quad m=1, \ldots, 3 \\
& \Delta \tau_{1}=0
\end{array}
$$

The constraint $(8 b)$ defines the coordinate system using the matrices

$$
A_{1}=\left[\begin{array}{lll}
e_{1} & e_{2} & e_{3}
\end{array}\right]^{T}, \quad A_{2}=\left[\begin{array}{ll}
e_{2} & e_{3}
\end{array}\right]^{T}, \quad A_{3}=e_{3}^{T},
$$

where $\left\{\boldsymbol{e}_{i}\right\}_{i=1}^{3}$ is the standard basis for $\mathbb{R}^{3}$. To simplify notation in the remainder of this paper we write (8) more compactly as

$$
\begin{array}{cl}
\min _{\boldsymbol{\theta}} & \frac{1}{2}\|\boldsymbol{\epsilon}(\boldsymbol{\theta})\|_{2}^{2} \\
\text { s.t. } & A \boldsymbol{\theta}=\boldsymbol{b}
\end{array}
$$

where we have introduced the stacked normalized residual vector $\boldsymbol{\epsilon}(\boldsymbol{\theta})$ and collected the constraints (8b) and (8c) in the linear system $A \boldsymbol{\theta}=b$, with $A$ and $b$ chosen accordingly.

Since our proposed calibration method does not require any surveying of positions, there are no restrictions on the size of the dataset and it becomes feasible to collect a dataset with a large number $(N \gg M)$ of transmitters. Furthermore, we choose to work with one transmission per transmitter, i.e., $K=1$. Such a dataset of $N$ stationary transmitters, each transmitting a single pulse, is equivalent to that of a single transmitter transmitting $N$ pulses at different positions. That is, the data collection procedure merely consists of moving a single transmitter in the measurement volume. This yields an efficient and simple procedure to collect a big dataset in little time.

\section{Solvers}

The optimization problem (10) is a nonlinear least squares problem with equality constraints. This class of problems is a well-known topic in optimization literature for which standard algorithms exist [8, 9], including (infeasible start) Newton methods and trust region methods.

Most optimization methods can be interpreted in the following sense. Given an initial guess, a local approximation is made. By solving this local problem a search direction is determined which is used to find a new and improved solution. The process is then repeated until convergence is obtained. For 
the problem under consideration, a common approximation is given by

$$
\begin{aligned}
\min _{\boldsymbol{\Delta} \boldsymbol{\theta}} & \frac{1}{2} \boldsymbol{\epsilon}^{T} \boldsymbol{\epsilon}+\boldsymbol{\epsilon}^{T} J \boldsymbol{\Delta} \boldsymbol{\theta}+\frac{1}{2} \boldsymbol{\Delta} \boldsymbol{\theta}^{T} J^{T} J \boldsymbol{\Delta} \boldsymbol{\theta} \\
\text { s.t. } & A \boldsymbol{\Delta} \boldsymbol{\theta}=\boldsymbol{b}-A \boldsymbol{\theta}
\end{aligned}
$$

where $J=\mathrm{D}_{\theta} \epsilon$ denotes the gradient of the normalized residuals with respect to the parameter $\boldsymbol{\theta}$. Note that all quantities are evaluated at $\boldsymbol{\theta}$. For this problem, the first order optimality (KKT) conditions $[8,9]$, can be written as

$$
\underbrace{\left[\begin{array}{cc}
J^{T} J & A^{T} \\
A & 0
\end{array}\right]}_{\triangleq \mathcal{K}}\left(\begin{array}{c}
\boldsymbol{\Delta} \boldsymbol{\theta} \\
\boldsymbol{\nu}
\end{array}\right)=\left(\begin{array}{c}
-J^{T} \boldsymbol{\epsilon} \\
\boldsymbol{b}-A \boldsymbol{\theta}
\end{array}\right)
$$

with $\nu$ the dual variable associated with the constraint (11b). Solving for $(\boldsymbol{\Delta} \boldsymbol{\theta}, \boldsymbol{\nu})$ in (12) yields a primal-dual search direction, which can be used in combination with an appropriately chosen step-size $s$ to update the solution as $\boldsymbol{\theta}:=\boldsymbol{\theta}+s \boldsymbol{\Delta} \boldsymbol{\theta}$.

The problem under consideration (11) is very sparse and contains a lot of structure. By exploiting this structure with for instance a sparse LDL-factorization or by applying a blockmatrix solver in combination with a QR-factorization of $J$, efficient implementations are obtained.

At the optimum, the KKT conditions (12) can be used to obtain the following gradient

$$
\mathrm{D}_{\boldsymbol{\epsilon}} \boldsymbol{\theta}=\mathrm{D}_{\boldsymbol{\epsilon}} \boldsymbol{\Delta} \boldsymbol{\theta}=-\left(\mathcal{K}^{-1}\right)_{11} J^{T} .
$$

Now, application of Gauss' approximation formula [10], in combination with the fact that normalized residuals have $\operatorname{Cov}(\epsilon)=I$, yields

$$
\begin{aligned}
\operatorname{Cov}(\boldsymbol{\theta}) & =\left[\mathrm{D}_{\boldsymbol{\epsilon}} \boldsymbol{\theta}\right] \operatorname{Cov}(\epsilon)\left[\mathrm{D}_{\boldsymbol{\epsilon}} \boldsymbol{\theta}\right]^{T} \\
& =\left(\mathcal{K}^{-1}\right)_{11} J^{T} J\left(\mathcal{K}^{-1}\right)_{11}=\left(\mathcal{K}^{-1}\right)_{11} .
\end{aligned}
$$

The last equality can be shown by expanding the $(1,1)$-block of $\mathcal{K}^{-1}$ as

$$
\begin{aligned}
\left(\mathcal{K}^{-1}\right)_{11} & =[I-X]\left(J^{T} J\right)^{-1}, \\
X & =\left(J^{T} J\right)^{-1} A^{T}\left(A\left(J^{T} J\right)^{-1} A^{T}\right)^{-1} A .
\end{aligned}
$$

Note that (15) shows that the covariance of the constrained problem is closely related to $\left(J^{T} J\right)^{-1}$, which is the covariance of the unconstrained problem.

As the size of the problem increases, the inversion of $\mathcal{K}$ can become infeasible. However, it is still possible to efficiently calculate parts of it. First, factor $\mathcal{K}$ as

$$
\mathcal{K}=L D L^{T}
$$

using a LDL-decomposition. Note that $L$ is a lower triangular matrix with unit diagonal, and $D$ is a block diagonal matrix consisting of $1 \times 1$ and $2 \times 2$ blocks. Rearranging (16), we have

$$
L^{T} \mathcal{K}^{-1}=D^{-1} L^{-1},
$$

where the lower triangular part of $D^{-1} L^{-1}$ is easily calculated. Hence, we can apply the method described in $[11,12]$ to efficiently calculate the elements of $\mathcal{K}^{-1}$ for which $L$ or $D$ are nonzero.

\section{Starting point}

The solvers described in the previous section can efficiently solve (8), but require a reasonable initial estimate to converge to the correct optimum since the problem is non-convex. Our approach is to find a starting point for (8) by solving a series of optimization problems.

The first step is to collect a dataset using $M$ transmitters that are placed in close proximity of the $M$ receivers. This implies that $\boldsymbol{r}_{m} \approx \boldsymbol{t}_{m}$, which allows us to extend (8) with this additional information and solve

$$
\begin{array}{ll}
\min _{\boldsymbol{\theta}} & \frac{1}{2} \sum_{m=1}^{M} \sum_{n=1}^{M} \sum_{k=1}^{K} \epsilon_{m n k}^{2}(\boldsymbol{\theta}) \\
\text { s.t. } & A_{m} \boldsymbol{r}_{m}=\mathbf{0} \quad m=1, \ldots, 3 \\
\Delta \tau_{1}=0 & \\
\boldsymbol{r}_{m}-\boldsymbol{t}_{m} & =\mathbf{0} \quad m=1, \ldots, M
\end{array}
$$

Solving this problem also requires a starting point, but when started in an arbitrary disjoint receiver configuration, i.e., $\boldsymbol{t}_{i} \neq \boldsymbol{t}_{j}$, it converges to the correct configuration or a (partially) mirrored version. To prevent the latter from happening, we start the optimization (18) given an approximate receiver configuration - a noisy, rotated and scaled version of the actual set of receiver positions.

The calibration parameters $\left\{\boldsymbol{r}_{m}, \Delta \tau_{m}\right\}_{m=1}^{M}$ obtained from solving (18) are biased, since the constraint (18d) only hold approximately. However, they provide a viable starting point for solving (8), and they can be used in (2) to determine initial values for the transmitter parameters $\left\{\boldsymbol{t}_{n}, \tau_{n}\right\}_{n=1}^{N}$. With this approach, a good starting point for (8) has been specified and the methods described in Section III-C can be used to solve it.

\section{EXPERIMENTAL RESULTS}

The proposed calibration method, summarized in Algorithm 1, has been used to calibrate a UWB setup (TimeDomain Plus hardware) in a relatively large volume, approximately

\section{Algorithm 1 UWB calibration}

1) Construct a setup consisting of $M$ stationary receivers.

2) Place $M$ transmitters in close proximity to the receiver antennas and collect a dataset $\mathcal{D}_{1}=\left\{y_{m n k}\right\}$.

3) Solve (18) for $\mathcal{D}_{1}$ to obtain $\left\{\boldsymbol{r}_{m}^{0}, \Delta \tau_{m}^{0}\right\}_{m=1}^{M}$. The optimization is started in

$$
\boldsymbol{\theta}^{0}=\left(\left\{\tilde{\boldsymbol{r}}_{n},\{0\}_{k=1}^{K}\right\}_{n=1}^{M},\left\{\tilde{\boldsymbol{r}}_{m}, 0\right\}_{m=1}^{M}\right)
$$

where $\left\{\tilde{\boldsymbol{r}}_{m}\right\}_{m=1}^{M}$ is a noisy, scaled and rotated version of the actual set of receiver positions.

4) Collect a second dataset $\mathcal{D}_{2}=\left\{y_{m n k}\right\}$ while moving a single transmitter through the measurement volume.

5) Solve (2) on subsets of $\mathcal{D}_{2}$ using the calibration values of Step 3 to obtain $\left\{\boldsymbol{t}_{n}^{0}, \tau_{n}^{0}\right\}_{n=1}^{N}$.

6) Solve (8) for $\mathcal{D}_{2}$. The optimization is started in

$$
\boldsymbol{\theta}^{0}=\left(\left\{\boldsymbol{t}_{n}^{0}, \tau_{n}^{0}\right\}_{n=1}^{N},\left\{\boldsymbol{r}_{m}^{0}, \Delta \tau_{m}^{0}\right\}_{m=1}^{M}\right),
$$

using the results from Step 3 and Step 5.

7) Calculate the calibration accuracy using (14). 


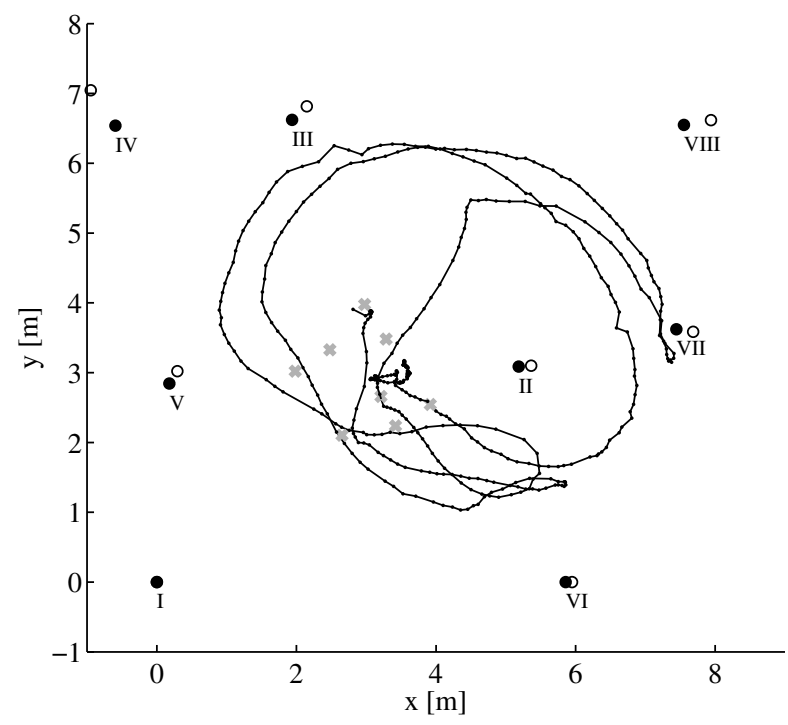

Fig. 1. Top view of the receiver locations and the transmitter trajectory of $\mathcal{D}_{2}$. We show the receiver configuration specified by the user $\tilde{\boldsymbol{r}}_{m}(\times)$, the initial receiver positions $\boldsymbol{r}_{m}^{0}(\mathrm{O})$, the calibrated receiver positions $\boldsymbol{r}_{m}(\bullet)$ and the transmitter trajectory $\boldsymbol{t}_{n}(-)$.
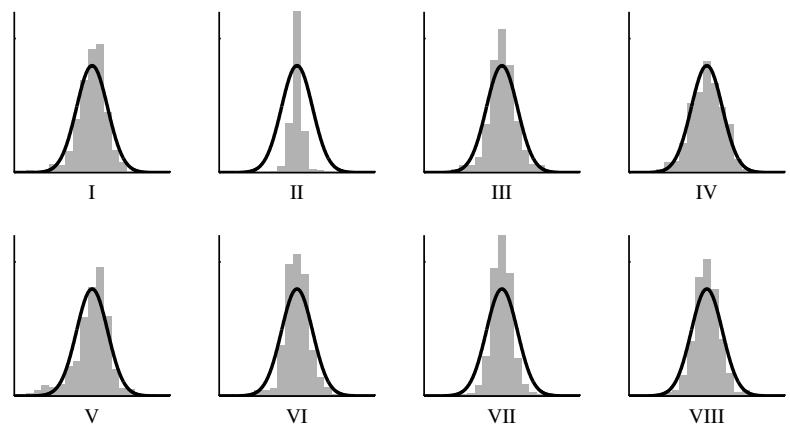

VII

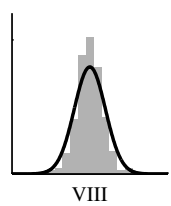

Fig. 2. Normalized residuals $\epsilon$ of $\mathcal{D}_{2}$ for each receiver after calibration Both the empirical distribution (bar) and the theoretical distribution (line) are shown.

$8 \times 8 \times 2.5 \mathrm{~m}$ in size. The setup consists of a total of 8 synchronized UWB receivers, 7 are attached to the ceiling and one is placed on the floor. Each receiver has a $1 \mathrm{~Hz}$ transmitter attached to its housing. A $10 \mathrm{~Hz}$ transmitter is moved through the measurement volume.

In this section we present the calibration results by applying Algorithm 1 to a $6 \mathrm{~s}$ dataset of the transmitters attached to the receivers $\left(\mathcal{D}_{1}\right)$ and a $50 \mathrm{~s}$ dataset where a transmitter is moved through the measurement volume $\left(\mathcal{D}_{2}\right)$. Figure 1 shows the receiver positions at various stages in the algorithm together with the transmitter trajectory. The approximate receiver configuration $\tilde{\boldsymbol{r}}_{m}$ used in Step 3 of Algorithm 1 clearly has the wrong scale and is rotated approximately $45^{\circ}$. Nevertheless, the approximate receiver positions $\boldsymbol{r}_{m}^{0}$ obtained provide a viable initial guess. Furthermore, the calibrated positions $\boldsymbol{r}_{m}$ do agree very well with their surveyed positions.

In order to further validate the calibration results, the normalized residuals $\epsilon$ of the moving transmitter dataset $\mathcal{D}_{2}$ are studied. The normalized residuals of $\mathcal{D}_{2}$ after Step 5 of Algorithm 1, that is using the initial guess $\left\{\boldsymbol{r}_{m}^{0}, \Delta \tau_{m}^{0}\right\}_{m=1}^{M}$, turn out (not shown) to be biased, but are reasonably close to their theoretical distribution. This indicates that the initial calibration values from (18) indeed are a viable starting point. Figure 2 shows the normalized residuals of $\mathcal{D}_{2}$ after Step 6 of Algorithm 1, using the calibrated values $\left\{\boldsymbol{r}_{m}, \Delta \tau_{m}\right\}_{m=1}^{M}$. Notice that the residuals are unbiased and that their distribution is close to that of white noise. The exception is receiver II, the only receiver on the floor. Because of the geometry of the setup, this receiver is very dominant in the determination of the height of the transmitter. Hence, the optimization can reduce its residuals without additional costs by slightly adjusting the height of the transmitter. The observation that the normalized residuals after calibration are close to the theoretical distribution implies that the model, with the estimated parameters and its assumptions, appears to be correct. This is in turn a very good indication that correct calibration parameters have been obtained.

\section{CONCLUSiON}

In this paper a novel calibration method is proposed to determine the clock parameters of the UWB receivers as well as their 3D positions. It exclusively uses time-of-arrival measurements, thereby removing the need for the typically labor-intensive and time-consuming process of surveying the receiver positions. Experiments show that the method is capable of accurately calibrating a UWB setup within minutes.

\section{ACKNOWLEDGMENT}

This work was partly supported by CADICS, a Linnaeus center funded by the Swedish Research Council.

\section{REFERENCES}

[1] Time Domain, 2010. [Online]. Available: http://www. timedomain.com/

[2] Ubisense, 2010. [Online]. Available: http://www.ubisense.net/

[3] Y. Chan and K. Ho, "A simple and efficient estimator for hyperbolic location," IEEE Trans. Signal Process., vol. 42, no. 8, pp. 1905-1915, Aug. 1994.

[4] S. Gezici, Z. Tian, G. Giannakis, H. Kobayashi, A. Molisch, H. Poor, and Z. Sahinoglu, "Localization via ultra-wideband radios: a look at positioning aspects for future sensor networks," IEEE Signal Process. Mag., vol. 22, no. 4, pp. 70-84, Jul. 2005.

[5] A. Sayed, A. Tarighat, and N. Khajehnouri, "Network-based wireless location: challenges faced in developing techniques for accurate wireless location information," IEEE Signal Process. Mag., vol. 22, no. 4, pp. 24-40, Jul. 2005.

[6] Z. Sahinoglu, S. Gezici, and I. Güvenc, Ultra-wideband Positioning Systems. Cambridge University Press, 2008.

[7] P. Misra and P. Enge, Global Positioning System: Signals, Measurements, and Performance, 2nd ed. Lincoln, MA, USA: Ganga-Jamuna Press, 2006.

[8] J. Nocedal and S. J. Wright, Numerical optimization, 2nd ed. New York: Springer-Verlag, 2006.

[9] S. Boyd and L. Vandenberghe, Convex Optimization. Cambridge University Press, 2004.

[10] L. Ljung, System Identification: Theory for the User, 2nd ed. Upper Saddle River, NJ, USA: Prentice-Hall, Inc, 1999.

[11] Å. Björk, Numerical Methods for Least Squares Problems. Philadelphia: SIAM, 1996

[12] G. H. Golub and R. J. Plemmons, "Large-scale geodetic leastsquares adjustment by dissection and orthogonal decomposition," Lin. Alg. Appl., vol. 34, pp. 3-28, dec 1980. 